\title{
Assessment and Management of Long COVID
}

\author{
Rafael A. Rivas-Vazquez · Gustavo Rey · Alan Quintana · Ana A. Rivas-Vazquez
}

Published online: 9 February 2022

(c) National Register of Health Service Psychologists 2022, corrected publication 2022

\begin{abstract}
Almost two years into the pandemic, the scientific and healthcare communities continue to learn a great deal regarding COVID-19, the disease produced by the severe acute respiratory syndrome coronavirus 2 (SARS-CoV-2). Broad variability during acute COVID-19 infection is seen, ranging from asymptomatic presentation to death. The vast majority of individuals who develop COVID-19 return to their pre-COVID-19 baseline within several weeks. However, a portion of patients will develop a post-COVID-19 syndrome of persistent cognitive, somatic, and behavioral symptoms. This syndrome, designated as post-acute sequelae of SARS-CoV-2 infection, is more commonly known as long COVID. The objectives of this paper are to inform psychologists regarding our current understanding of the underlying pathophysiology of COVID-19, review criteria for range of severity during acute illness, present clinical manifestations of long haul phenomena, and discuss the emerging literature base of evidence-based treatment and management approaches.
\end{abstract}

Key words COVID-19 $\cdot$ PASC $\cdot$ Long haulers $\cdot$ Long COVID

\section{Clinical Vignettes}

Antonio, a 52-year-old attorney with hypertension and diabetes mellitus, developed fever, cough, and shortness of breath. Upon presentation to the emergency room, he tested positive for SARS-CoV-2. He was noted to have pneumonia, low oxygen saturation $(<85 \%)$, and was initially given highflow canula oxygen, but went on to develop acute respiratory distress syndrome (ARDS) and demonstrate altered mental status, requiring intubation. He subsequently developed acute renal failure and was placed on dialysis and anticoagulation therapy. He was eventually stabilized and discharged after eight weeks. He presented to our clinic with symptoms of cognitive impairment and possible posttraumatic stress disorder.

Sandra is a 21-year-old collegiate athlete in excellent physical health. After developing headaches, chills, and diminished sense of smell and taste, she was found to be COVID-19 positive. In compliance with university protocol, she quarantined for two weeks and was cleared to return to the classroom and practice after two consecutive negative PCR tests. She presented to our clinic four months later with fatigue, persistent headache, shortness of breath upon exertion, and difficulty focusing and staying on task. She was referred by her coach for assessment due to difficulty in the classroom and inability to resume her premorbid level of athletic activity.

Timothy, a 28-year-old waiter in good health, underwent testing for COVID-19 after his wife developed respiratory symptoms and tested COVID-19 positive. He was fully asymptomatic, but tested PCR+ and underwent a 10-day quarantine, eventually testing negative and returning to work. Approximately four weeks later, he developed malaise, myalgia, fatigue, and short-term memory difficulty. He presented to our clinic with anxiety about his health status and concern that his employer thought he was feigning symptoms.

\section{Background and Challenges}

Almost two years into this historic pandemic, the scientific and healthcare communities continue to learn a great deal regarding COVID-19, the disease that is produced by the severe acute respiratory syndrome coronavirus 2 (SARS-CoV-2). As of 2021, an estimated 249 million cases of COVID-19 were confirmed, including more than 5 million deaths (World Health Organization 
[WHO], 2021a). The disease presents with a broad range of clinical variability, from individuals who are asymptomatic following exposure to SARS-CoV-2 to those who develop severe and critical illness that may ultimately prove fatal. Although the most common and acute presentations involve respiratory compromise, central nervous system (CNS) involvement-through direct and indirect mechanisms-has also been seen.

The most urgent and immediate focus has been placed on identifying and developing effective therapeutic interventions during the acute phase of the illness; however, recent attention has increasingly been drawn to residual symptoms manifested by those who are post-COVID-19. While the majority of individuals infected with SARS-CoV-2 will return to their premorbid baseline within several weeks, it is now known that some individuals will have persistent cognitive, somatic, and behavioral symptoms (CSBS) that may last up to several months, possibly a year. This protracted syndrome is being referred to as "long COVID," and individuals manifesting this persistent state have been designated as "long haulers" (Collins, 2021).

Psychologists will increasingly be called upon to assess individuals presenting with these complaints following COVID-19. The objectives of this paper are to discuss pathophysiological mechanisms suspected to mediate CNS dysfunction, to review current criteria to determine acute COVID-19 disease severity, and to present what is currently known regarding the phenomena of long COVID and potential management approaches.

\section{Pathophysiology Underlying CNS Dysfunction}

The severity of COVID-19 that each individual demonstrates during the acute phase is directly related to the degree of the body's immune response. This response will in turn be determined by a host of factors including age, genetic predisposition, immune system status, prior exposure to homologous pathogens, and the presence of any comorbid conditions (e.g., cardiovascular disease, chronic lung disease, diabetes, cancer, obesity) that may render those particular systems more vulnerable to dysfunction.

The life cycle of COVID-19 follows a distinct process (Yuki et al., 2020). Once an individual is exposed to SARS$\mathrm{CoV}-2$, the virus attaches to host receptors and penetrates host cells (either through endocytosis or membrane fusion). Following penetration, viral contents are released inside the host cell and viral RNA enters the nucleus to alter normal transcription. Viral proteins are then synthesized, reach maturation, and are released. CoVs consist of four structural proteins, including a spike $(\mathrm{S})$ protein that binds to angiotensin converting enzyme 2 (ACE2; Chen et al., 2020).

ACE2 is present on the surface of various cell types and abundant in heart, ileum, kidney, bladder, and lung, where it is highly expressed in epithelial cells (which serve as protective surface barriers for blood vessels and organs). ACE2 plays a role in regulating cellular processes, primarily by modulating the activity of angiotensin II, a vasoconstrictor protein that increases blood pressure and inflammation. By binding to ACE2, SARS-CoV-2 not only gains entry into the cell but prevents ACE2 from breaking down angiotensin II. Toxic over-accumulation of plasma angiotensin II contributes to cellular injury to the heart and lungs, potentially inducing myocarditis (i.e., inflammation of the heart muscle) and acute respiratory distress syndrome (Hanff et al., 2020).

Once the virus is detected in the body, our natural immune response triggers a cascade of events resulting in production of antiviral interferons and cytokines (i.e., signaling proteins that trigger the protective defenses of the immune system). It is now thought that SARS-CoV-2 may induce a particular signature response characterized by induction of multiple pro-inflammatory cytokines (García, 2020); this response may initiate a progression from a typically adaptive immune response to a potentially more damaging and exaggerated inflammatory reaction (Tay et al., 2020). This severely acute systemic inflammation has been referred to as a "cytokine storm," and is thought to underlie the prevalence of mortality associated with COVID-19 (Tang et al., 2020).

Furthermore, emerging evidence suggests that SARS$\mathrm{CoV}-2$ may produce prolonged inflammation even after the virus is no longer active and detectable on testing, a characteristic that may explain why symptoms are persistent and come in waves after the acute phase of illness has concluded (Davido et al., 2020; Schmidt, 2021). The cytokine storm may serve as the initiating event that leads to potentially broad pathological reactions across various organ systems, which in turn can directly and indirectly compromise the CNS and contribute to long COVID.

CNS dysfunction, in both the acute and residual phase, can be understood in terms of inflammation, de-oxygenation, coagulation, and possible neurotransmitter dysregulation. SARS-CoV-2 can produce direct inflammation of the brain and meninges (Inouye, 2021), resulting in edema and encephalitis that can range in severity from mild and transient to catastrophic with persistent neurologic deficits. Beyond the CNS, the hyper-inflammatory response associated with a cytokine storm may contribute to dysfunction at other organ sites (e.g., kidneys, liver), indirectly compromising CNS functioning by producing metabolic dysregulation and altered mental status (i.e., metabolic encephalopathy).

Hypoxemia, defined as below-normal levels of oxygen in the blood, is also a critical factor mediating acute and residual CNS dysfunction. The human brain is one of the most metabolically active organs in the body, typically representing only $2 \%$ of total body weight, but requiring 
$20 \%$ of total body oxygen for normal functioning (Rink $\&$ Khanna, 2011). Consequently, the brain's high oxygen requirement means that its tissue is extremely sensitive to changes in arterial blood oxygen levels (Kumar, 2011). Taken as a whole, the exaggerated inflammatory process produced by SARS-CoV-2 within the lungs, the heart (Imazio et al., 2020), and the peripheral vascular system (Østergaard, 2021) can produce hypoxemia, leading to CNS anoxic/hypoxic injury.

Coagulopathy (i.e., impairment in the blood's clotting ability) is another element of the systemic inflammatory response in severe COVID-19. It can result in hemorrhagic (bleeding) and thrombotic (clotting) cerebrovascular events, which can compromise CNS functioning (Gómez-Mesa et al., 2021; Inouye, 2021; Leentjens et al., 2021). Finally, as a result of pathological mechanisms noted above (i.e., exaggerated inflammation and hypoxemia), it has been speculated that COVID-19 CNS dysfunction may also be mediated by neurotransmitter dysregulation-a finding that may allow us to better understand the persistence of symptoms seen in long COVID.

The synthesis of biogenic amines (e.g., dopamine, norepinephrine, epinephrine, serotonin) relies heavily on oxygen availability; consequently, hypoxemia can result in disruptions in neurotransmitter synthesis and availability, which contribute to altered neuronal function (Kumar, 2011). Furthermore, pro-inflammatory cytokines appear to alter the metabolic pathway of the amino acid tryptophan (typically metabolized into serotonin and melatonin) to produce neurotoxic levels of kynurenine, quinolinic acid, and glutamate. These changes may result in further neuronal damage and alterations in neurotransmission (Boldrini et al., 2021; Capuron \& Miller, 2011).

\section{Course and Severity of COVID-19}

The course of COVID-19 illness is divided into two main phases. The acute phase is when the individual begins to manifest symptoms and the presence of SARS-CoV-2 is confirmed (typically via positive polymerase chain reaction [PCR] test). This phase is followed by the recovery phase in which the individual has generated a negative PCR test, the acute phase symptoms begin to subside, and the individual begins experiencing a return to their premorbid baseline. It is the recovery phase that has been the recent source of much attention and discussion.

Following exposure and incubation, the acute phase of COVID-19 infection induces a range of symptom severity in individuals, from asymptomatic presentation following a positive PCR test result to critical illness requiring medical intervention (and with a potentially fatal outcome). The World Health Organization's current classification of COVID-19 infection severity has guidance for asymptomatic infection and mild, moderate, severe, and critical illness, of which health practitioners should be aware (COVID-19 Treatment Guidelines Panel, 2021).

Following exposure to SARS-CoV-2, the majority of individuals will go on to develop symptoms meeting criteria for mild to moderate COVID-19 illness. Approximately 20\% will develop more severe illness, with $15 \%$ requiring hospitalization and 5\% requiring ICU care. The overall mortality risk of COVID-19 is currently $0.5 \%$ to $1.0 \%$ (Auwaerter, 2021). Accurate assessment of residual CSBS that persists as an individual transitions into the recovery phase will be based on a thorough review and understanding of the severity of illness that the individual manifested during the acute phase.

\section{What Are Long Haul COVID-19 Phenomena?}

While the majority of individuals who develop symptomatic COVID-19 will return to their baseline within 2 to 3 weeks of resolution of acute symptoms, there are some who will have prolonged impairment (Aiyegbusi et al., 2021; Tenforde et al., 2020). Those who are most at risk for persistent CSBS are individuals who met criteria for severe-critical illness during acute COVID-19 and experienced CNS insult secondary to any of the pathological mechanisms discussed above. Cerebrovascular accident, vasculitis, delirium/ encephalopathy (altered mental status), encephalitis, seizure, hypoxic/ischemic injury, seizures, demyelinating lesions, and Guillain-Barré syndrome have all been reported in the setting of severe to critical acute COVID-19 (Frontera et al., 2021; Kotfis et al., 2020; Toscano et al., 2020; Varatharaj et al., 2020).

For example, Antonio, the first patient presented in our clinical vignettes, met criteria for severe illness and demonstrated altered mental status secondary to hypoxemia, which can result in persistent neurocognitive (e.g., cognitive slowing, executive dysfunction) and neurobehavioral (e.g., irritability, affective lability, disinhibition) sequelae. For individuals with severe to critical acute disease, postCOVID-19 management can involve a detailed review of medical history, events during active illness, and thorough diagnostic assessment to develop an appropriate treatment plan. This plan should address cognitive and psychological issues, including collaboration with other health care specialties (e.g., neurology, cardiology) to ensure a holistic intervention approach.

The more puzzling phenomena center around individuals who have had milder forms of acute COVID-19 and have prolonged impairment. One of the first accounts of "frightening and long" symptom persistence appeared in the literature on May 5, 2020, from Dr. Paul Garner, a British infectious disease professor who developed mild symptoms 
that did not require hospitalization. Written seven weeks following initial symptom onset, Dr. Garner described "a roller coaster of ill health, extreme emotions, and utter exhaustion" that involved not only symptom persistence, but waves of abating and resurging symptoms (Garner, 2020). He characterized this condition as the "long tail" of the disease; soon after, other terms such as "long COVID" began appearing in the literature.

The precise term "long hauler" was borne out of and popularized by the collective experience of individuals who were struggling with persistent post-COVID-19. The term was originally championed by Amy Watson, who was wearing a trucker hat when she was initially tested for SARS-CoV-2 and who went on to establish Long Haul COVID Fighters, a web-based support format that provides support for those with long COVID and serves as an advocacy mechanism for medical, psychological, and social needs of long haul COVID survivors. The Centers for Disease Control and Prevention has officially named long COVID "post-acute sequelae of SARS-CoV-2" (PASC) for research purposes (Collins, 2021).

Although no precise definition exists, long COVID is typically characterized as CSBS persisting four weeks after initial infection (Crook et al., 2021). Across various studies, the most common long COVID symptoms are fatigue, shortness of breath, myalgia (muscle pain), headache, decreased sense of smell or taste, post-exertional malaise, inability to return to exercise or normal activity levels, cognitive dysfunction, depression, apathy, anxiety, and possible posttraumatic stress symptoms (Aiyegbusi et al., 2021; Davis et al., 2020; Lambert et al., 2021; Logue et al., 2021; Taquet et al., 2021). It should be noted that some of the behavioral symptoms of long COVID speculated to be mediated by persistent inflammation (e.g., fatigue, apathy, lowered mood) may be indistinguishable from primary psychological symptoms of depression and anxiety that might reflect adjustment response to trauma and psychosocial hardship endured during COVID-19.

A lack of cognitive acuity has increasingly been described in the literature as "brain fog" (Couzin-Frankel, 2020; Graham et al., 2021; Rubin, 2020), a nonspecific term that has been used to characterize cognitive alterations associated with other neurologic and immunologic conditions (e.g., fibromyalgia, multiple sclerosis). Although there is not yet consensus on how to define this term, memory loss, poor focus, reduced concentration, increased word-finding latency, difficulty tracking complex information, and decreased executive functions have all been associated with the term. Lamontagne et al. (2021) assessed mood and cognitive symptoms in 50 healthy individuals (with no prior history of COVID-19 infection) versus 50 post-COVID-19 individuals. The two groups did not differ with respect to age, gender, education, race/ethnicity, or pandemic stress experiences. Participants received assessments for stress, depression, attention, orienting, and executive functions. Results indicated significantly higher levels of depression, anhedonia, inattention, and executive dysfunction in the post-COVID-19 group, with the most pronounced effects being noted in individuals who were 1 to 4 months postacute phase (Lamontagne et al., 2021).

The exact prevalence of long COVID has been difficult to specify, in part due to differences in follow-up examination intervals, as well as lack of consensus on number of symptoms required to meet criteria for long COVID (WHO, 2021b). Taquet et al. (2021) conducted a retrospective review of 236,379 electronic health records of COVID-19 recovered patients, $80 \%(190,077)$ of which had mild illness. At six-month follow-up, 57\% of their sample had at least one or more long COVID symptom. Other studies have reported rates of long COVID symptoms of $87 \%$ at two months, $96 \%$ at three months, and $76 \%$ at six months (Carfì et al., 2020; Davis et al., 2020; Huang et al., 2021b). Logue et al. (2021) reported on a prospective nine-month follow-up study with 177 post-COVID-19 individuals, 161 (91\%) of which had had asymptomatic or mild acute illness. At follow-up, $30 \%$ of their sample reported persistent long COVID symptoms, the most common of which was fatigue. Many long haulers also state that their symptoms come in waves. Individuals in this subgroup state that their initial symptoms, which may have been respiratory or cardiac in nature, dissipated during initial recovery, only to be followed a brief time later by a second (and possibly a third) wave of other CSBS (Davis et al., 2020; Lambert et al., 2021).

There is also interest in attempting to predict who will become long haulers. Female sex and severity of illness during acute COVID-19 (specifically, the presence of $>$ 5 symptoms; Aiyegbusi et al., 2021) are associated with increased likelihood of reporting long COVID symptoms. With regard to symptomatology during acute COVID-19, however, it should be noted that a "symptom lag" has been described in a subset of patients who go on to manifest long COVID symptoms (Logue et al., 2021). These individuals were either asymptomatic or very mild (reporting only minimal respiratory symptoms) during COVID-19 positive phase, and then began experiencing new-onset CSBS after an interval of having tested negative. Huang et al. (2021a) reviewed records of 1,407 post-COVID-19+ individuals for presence of symptoms $\geq 60$ days following initial PCR positive testing. They found that $32 \%$ of individuals reporting symptoms after a period of a least two months post-COVID were asymptomatic during the acute phase.

\section{Mechanisms of Long COVID}

The underlying mechanism of long COVID is not fully understood. Specifically, the link between long-haul 
phenomena and purported pathophysiological elements of acute COVID-19 (e.g., hyper-inflammation, coagulation, hypo-oxygenation, and possible neurotransmitter dysregulation) are unknown. The persistence of symptoms long after individuals have tested negative for COVID-19, coupled by observations of symptom lag and symptom waves, has drawn speculation as to whether the body's immune response becomes aberrantly and intermittently activated even after there is no longer detectable virus in the body (Huang et al., 2021b).

Within the cardiac literature, there have been findings of persistent myocardial inflammation and cardiac dysfunction (demonstrated by elevated cardiac enzymes and lower ejection fraction) up to 3 months following recovery from COVID-19 positive status (Puntmann et al., 2020). One theory is that an analogous inflammation-associated dysregulation is taking place within the CNS and resulting in the CSBS manifested by the long haulers (Cothran et al., 2020). Evidence supporting this hypothesis is provided by Patterson et al. (2021a), who found persistently elevated levels of intermediate and non-classical monocytes (white blood cells involved in the immune and inflammatory response; see Patterson et al., 2021a) in patients with long COVID versus normal controls up to 15 months post infection. Moreover, the profile of particular cytokine elevations, in addition to prolonged nature of the elevations, may be a signature element specific to PASC (Patterson, 2021b).

Functional imaging data using fluorodeoxyglucose (FDG)-positron emission tomography (PET), which is an established biomarker of CNS inflammation, may serve to elucidate the mechanisms underlying persistent clinical manifestations reported by long haulers. FDG PET performed on a small series $(\mathrm{N}=15)$ of recovered patients revealed pathological results in 66\% (10/15) of this sample, characterized by frontotemporal hypometabolism (Hosp et al., 2021). This finding correlated with impaired performance on the Montreal Cognitive Assessment (Nasreddine et al., 2005) prompted the authors to theorize that FDG PET may serve as a target for rehabilitative intervention. It should be noted that this study involved patients who had more severe acute illness requiring hospitalization. It remains to be seen what future functional imaging studies with patients recovering from mild acute phase illness will demonstrate.

Comparisons are also being drawn between fibromyalgia and myalgic encephalomyelitis/chronic fatigue syndrome (ME/CFS), with the suspected mechanism involving mediation by pro-inflammatory cytokines involved in long COVID (Komaroff, 2020; Moghimi et al., 2021). These recent findings pertaining to long COVID are in keeping with prior work by Reichenberg et al. (2001) who induced a depressive-like state in healthy volunteers by infusing them with a low-dose endotoxin known to trigger the release of cytokines. It is also supported by prior evidence that depression is associated with a chronic-low grade inflammatory response (Berk et al., 2013). Pitharouli et al. (2021) conducted a case-controlled study of 26,894 participants with major depression and found that their levels of C-reactive protein, which serves as a marker for inflammation, was significantly higher relative to nondepressed controls. Concurrently, there is evidence that antidepressants may modulate the inflammatory response by suppressing the production of pro-inflammatory cytokines (Berk et al., 2013; Maes et al., 1999).

Based upon this suspected mechanism, Lenze et al. (2020) randomly assigned 152 COVID-19 positive non-hospitalized patients to either the selective serotonin reuptake inhibitor fluvoxamine or to a placebo. Over a span of 15 days, the patients treated with fluvoxamine had a lower likelihood of clinical deterioration compared to the placebo group. Similarly, in patients with more severe illness, Hoertel et al. (2021) conducted a retrospective chart review of 7,230 adults hospitalized for COVID-19 and found that those who received an antidepressant within 48 hours of admission demonstrated lower risk of death or intubation, independent of clinical and biological markers of illness severity. Taken together, it seems reasonable to suspect persistent inflammation within the CNS, myocardium, lungs, and musculature as a critical element mediating the CSBS profile reported by long haulers.

It is important to note the limitations inherent in reports that have appeared to date in the literature regarding long COVID, which have been largely observational in nature. We await results from prospective, well-controlled clinical trials that will allow for more definitive information regarding the mechanisms linking exposure to SARS-CoV-2 and development of long COVID. As psychologists treating patients with long COVID, mechanisms pertaining to mental health components and related sequelae will be of particular relevance in developing targeted assessments and treatments for long COVID.

\section{Proposed Recommendations for Management of Long COVID}

As time elapses, the scientific and healthcare communities are continuously expanding their knowledge base about long COVID. Clinical and empirical data continue to amass regarding its pathophysiology, symptomatic presentation, course of illness, specific risk factors, and effective interventions. One of the unanswered and concerning questions involves ultimate time to full recovery. Specifically, what does recovery look like for a subset of individuals who are exposed to SARS-CoV-2 and go on to manifest a chronic and unremitting post-COVID-19 syndrome? The troubling parallel has been drawn to individuals who develop post-infection 
ME/CFS and who have difficulty fully recovering or returning to their premorbid level of health (Moghimi et al., 2021), raising this as a potentially unfavorable outcome for some COVID-19 long haulers.

Given the many unanswered questions, there has been a global call for recognition of long COVID as an accepted clinical syndrome. The World Health Organization has urged countries to increase their efforts to systematically collect post-COVID-19 data and to prioritize therapeutic and rehabilitative development for those struggling with long COVID. In the United States, Congress recently appropriated 1.15 billion dollars in funding over four years to the National Institutes of Health to support research identifying clinical and demographic variables that may serve as risk factors for development of long COVID, as well as elucidating the prolonged health consequences for post-COVID-19 individuals who are manifesting this syndrome (Collins, 2021).

To date, there has not been enough time to empirically develop, assess, and identify efficacious interventions for long COVID. Pending more definitive data, recommendations have been derived from experiences with other viral or bacterial infectious diseases (e.g., Epstein-Barr virus, Lyme disease) or extrapolated from other events producing CNS insult (e.g., stroke, anoxia/hypoxia, traumatic brain injury).

\section{Initial Assessment}

Appropriate management begins with thorough assessment of an individual's clinical and functional status. In the current healthcare environment, psychologists should be inquiring about known or possible SARS-CoV-2 exposure when conducting consultations with all new patients. For individuals who were COVID-19 positive, a review of medical history is critical to categorize the severity of the acute phase according to criteria listed above; specifically, whether they required hospitalization or oxygen support. As previously noted, individuals with severe-to-critical acute illness are at greater risk for CNS insult secondary to various mechanisms (i.e., stroke, encephalitis, hypoxic/anoxic injury). Aside from neurobehavioral manifestations of direct CNS injury, these individuals are also at psychological risk for developing depression, anxiety, and posttraumatic stress disorder secondary to trauma experienced during their hospitalization, as well as persistent physical and cognitive limitations during recovery.

In the case of Antonio, the 52-year-old attorney with severe COVID-19 from our clinical vignettes, he manifested altered mental status as a sign of hypoxic encephalopathy (or delirium), which places him at risk for persistent cognitive sequela that will require thorough assessment. Furthermore, he developed posttraumatic stress phenomena centered around being intubated and his fear of dying. He will consequently require appropriate treatment targeting this symptomatology.

In addition to data routinely collected in a comprehensive history of present illness, psychologists should systematically review for the presence of CSBS typically reported by long haulers, explicitly characterize any pre- and postCOVID-19 changes, and assess for the presence of any functional limitation (i.e., inability or inefficiency in performing at pre-COVID-19 vocational, academic, and recreational levels) that may serve as targets for rehabilitative efforts. As noted in the case of Sandra, the 21-year-old collegiate athlete with mild COVID-19 from our clinical vignettes, she was referred due to difficulties in resuming her pre-COVID-19 capacity to study and focus in the classroom, as well as perform at her characteristic level on the playing field. As long haulers return to work, they oftentimes describe diminished ability in managing their typical workload. More recently, we have encountered post-COVID-19 patients who are sufficiently concerned regarding difficulty in resuming their capacity to function at work that they have begun exploring the process for seeking disability benefits (which will add a level of complexity when assessing individuals reporting long haul phenomena). Whenever available, history regarding clinical symptoms and functional status should also be obtained from a collateral informant.

\section{Considerations for Underrepresented Individuals}

Finally, attention to ethnic and racial factors that may be impacting course of illness and access to care is critical during the assessment process. Since the early stages of the pandemic, racial disparities were identified and became a central topic of discussion at social and governmental levels. While these disparities have existed for generations, COVID-19 has created heightened awareness of the influence of socioeconomic factors in health outcomes. Underrepresented groups have been disproportionately affected by COVID-19 with a much higher incidence of disease, hospitalization, and death (Lopez et al., 2021). Medical comorbidities, environmental factors, and reduced access to health care have been identified as contributing variables in these epidemiological differences.

Yehia et al. (2020) examined mortality rates in 11,210 Black and White patients with confirmed SARS-Cov-2 infection requiring hospitalization due to severe ARDS. After adjusting for age, sex, insurance status, comorbidities, neighborhood deprivation, and site of care, there was no statistically significant difference in risk of mortality between groups. The authors concluded that sociodemographic factors and comorbidities, which may be contributing to race-related factors in disease outcome, can be reduced by ensuring appropriate access to care. Although the urgency of addressing these disparities has been recognized, the success in diminishing these inequities will require challenging 
transformations in health care and governmental policies, including expanding access to medical services and development of a more culturally equitable care model (Boulware, 2020; Lopez et al., 2021).

\section{Collaborating With Other Specialties}

Many post-COVID-19 patients, particularly those with more severe acute illness, will require a multidisciplinary approach. Following thorough initial assessment to characterize their CSBS profile, appropriate long COVID management may involve referral to other therapeutic modalities (e.g., speech, occupational, physical therapy) and collaboration with other health care specialties (e.g., neurology, cardiology, pulmonary). Our experience is that many patients will benefit from formal neuropsychological assessment to better characterize and document their post-COVID-19 cognitive status.

Given the significant prevalence of depression, anxiety, apathy, and insomnia reported by post-COVID-19 patients, a critical element of thorough initial assessment is evaluation for behavioral symptoms of sufficient severity to warrant referral for psychopharmacological intervention. To date, there is little data regarding the role of specific psychotropic agents that may mitigate long COVID. Extrapolating from data from other CNS disorders, studies are already underway regarding the application of antidepressants, stimulants, and other wakefulness-promoting agents (e.g., modafinil) in managing post-COVID-19 CSBS profile. Based on the application of antidepressants administered to acute COVID-19 patients during hospitalization, Mazza et al. (2021) identified 60 individuals who had developed depression within six months of recovery from COVID19 and treated them with selective serotonin reuptake inhibitor antidepressants. After four weeks of treatment, 55 (92\%) patients had demonstrated a clinical response, as defined as a $50 \%$ reduction in depression scores. In the context of limitations posed by the observational design of the study and lack of control group of antidepressanttreated depressed patients who were not post-COVID-19 positive, the observed rapidity of response in this sample prompted the authors to speculate that an anti-inflammatory mechanism of action inherent in these antidepressants may have mediated their favorable clinical outcome. It should be noted that these data are preliminary but seem to be serving as the basis for empirical application of antidepressants to long COVID, pending more definitive data from controlled clinical trials that are currently underway.

\section{Providing Psychological Support}

The need for mental health services has clearly increased during the course of the pandemic. For many individuals never having tested positive for SARS-CoV-2, there has invariably been exposure to trauma and adverse psychosocial events, such as loss of loved ones, financial hardship, and protracted social isolation during the pandemic. Those individuals who were COVID-19 positive and who are now demonstrating long haul CSBS are a distinct group who stand to benefit from psychological support. Systematic data from studies of psychotherapeutic modalities specifically for long COVID are not yet available. To date, some data indicate that cognitive behavioral therapy (CBT) may not be efficacious as a stand-alone treatment for long COVID-19 fatigue (Vink \& Vink-Neise, 2020). Further research is warranted, however, to determine if CBT or other evidence-based practice may be helpful for mental health sequelae associated with long COVID-19.

In our experience, psychologists play a key role in the management of long COVID by providing education, validation, reassurance, support, and direction. Many afflicted individuals are still not aware of long-haul phenomena, and benefit from receiving basic education and a sense of reassurance that this is now a recognized syndrome (Collins, 2021). Sandra (21-year-old collegiate athlete) and Timothy (28-year-old waiter in good health) from our clinical vignettes voiced a sense of relief after initial consultation in which education and support were offered. Sandra's care involved referral to neurology for treatment of her headaches (and she was also started on SSRI). She benefitted from supportive therapy and assistance while resuming her premorbid level of activity. In this regard, to the extent that it is possible, a slow and gradual re-entry to the classroom and the work environment is recommended. Similar to phenomena observed with patients demonstrating post-concussion syndrome following head injury, rapid return to work or school can exacerbate cognitive and behavioral symptoms and prolong recovery. Timothy, who manifested a symptom lag in which he was asymptomatic during the active phase and developed CSBS four weeks after testing negative, reported diminished anxiety and worry after receiving education, validation, and support, as well as some advocacy on his behalf with his employer to confirm that what Timothy was describing was genuine and clinically plausible.

\section{Conclusion}

Long COVID is a recognized post-viral syndrome consisting of cognitive, somatic, and behavioral symptoms that may last several months or more following acute COVID19 illness. While the majority of individuals infected with SARS-CoV-2 will return to their premorbid baseline within several weeks, some individuals will have persistent long COVID symptoms, which can range from mildly distressing to significantly interfering with essential daily 
activities. There has been a global call to systematically collect data regarding long COVID and to identify and develop effective treatment and rehabilitative interventions. Psychologists are well positioned to provide education regarding long COVID and evidence-based therapeutic intervention with the objective of improving outcome.

\section{Key Points}

1. The majority of individuals infected with SARS-CoV-2 will return to their premorbid baseline within several weeks.

2. Long COVID is a recognized syndrome consisting of cognitive, somatic, and behavioral symptoms that may last several months or more.

3. Persistent long COVID symptoms can range from mildly distressing to significantly interfering with essential daily activities.

4. Effective treatment and rehabilitative interventions for long COVID are direly needed.

5. Psychologists are integral in providing the assessment and evidence-based care that is likely required to improve outcomes for those with long COVID.

\section{Disclosures}

Conflicts of interest We have no known conflicts of interest, external funding, or other disclosures to make.

\section{References}

Aiyegbusi, O.L., Hughes, S.E., Turner, G., Rivera, S.C., McMullan, C., Chandan, J.S., ... TLC Study Group. (2021). Symptoms, complications and management of long COVID: a review. Journal of the Royal Society of Medicine, 114(9), 428-442. Doi: https://doi.org/10.1177/01410768211032850

Auwaerter, P. G. (2021). Coronavirus COVID-19 (SARS-COV-2): Johns Hopkins Abx Guide. Coronavirus COVID-19 (SARSCoV-2) Johns Hopkins ABX Guide. Accessed November 17, 2021. https://www.hopkinsguides.com/hopkins/view/Johns_ Hopkins_ABX_Guide/540747/all/Coronavirus_COVID_19_ SARS_CoV_2

Berk, M., Williams, L.J., Jacka, F.N., O’Neil, A., Pasco, J.A., Moylan, S., ... Maes, M. (2013). So depression is an inflammatory disease, but where does the inflammation come from? Current Controversies in Psychiatry, 11:200. https://doi.org/10.1186/ 1741-7015-11-200

Boldrini, M., Canoll, P. D., \& Klein, R. S. (2021). How COVID-19 Affects the Brain. JAMA Psychiatry. https://doi.org/10.1001/ jamapsychiatry.2021.0500

Boulware, L.E. (2020). Race disparities in the COVID-19 pandemic - solutions lie in policy, not biology. JAMA Netw Open, 3(8):e2018696. doi:https://doi.org/10.1001/jamanetworkopen. 2020.18039
Capuron, L., \& Miller, A. H. (2011). Immune system to brain signaling: Neuropsychopharmacological implications. Pharmacology \& Therapeutics, 130(2), 226-238. https://doi.org/10.1016/j. pharmthera.2011.01.014

Carfì A, Bernabei R, Landi F; Gemelli Against COVID-19 PostAcute Care Study Group. (2020). Persistent symptoms in patients after acute COVID-19. JAMA. 11;324(6):603-605. doi: 10.1001/jama.2020.12603. PMID: 32644129; PMCID: PMC7349096.

Chen, Y., Guo, Y., Pan, Y., \& Zhao, Z. J. (2020). Structure analysis of the receptor binding of 2019-nCoV. Biochemical and Biophysical Research Communications, 525(1), 135-140. https:// doi.org/10.1016/j.bbrc.2020.02.071

Collins, F.S. (2021). NIH launches new initiative to study "Long COVID." National Institutes of Health (NIH). https://www.nih. gov/about-nih/who-we-are/nih-director/statements/nih-launc hes-new-initiative-study-long-covid.

Cothran, T. P., Kellman, S., Singh, S., Beck, J. S., Powell, K. J., Bolton, C. J., \& Tam, J. W. (2020). A brewing storm: The neuropsychological sequelae of hyperinflammation due to COVID19. Brain, Behavior, and Immunity, 88, 957-958. https://doi. org/10.1016/j.bbi.2020.06.008

Couzin-Frankel, J. (2020). The long haul. Science, 369(6504), 614617. https://doi.org/10.1126/science.369.6504.614

COVID-19 Treatment Guidelines Panel. (2021). Coronavirus Disease 2019 (COVID-19) Treatment Guidelines. National Institutes of Health. Available at https://www.covid19treatmentguidelines. nih.gov/. Accessed 5/13/2021

Crook, H., Raza, S., Nowell, J., Young, M., \& Edison, P. (2021). Long covid-mechanisms, risk factors, and management. British Medical Journal, 374, n1648. https://doi.org/10.1136/bmj. n1648

Davido, B., Seang, S., Tubiana, R., \& de Truchis, P. (2020). PostCOVID-19 chronic symptoms: a postinfectious entity? Clinical Microbiology and Infection, 26(11), 1448-1449. https://doi.org/ 10.1016/j.cmi.2020.07.028

Davis, H. E., Assaf, G. S., McCorkell, L., Wei, H., Low, R. J., Re'em, Y., ... Akrami, A. (2020). Characterizing long COVID in an international cohort: 7 months of symptoms and their impact. https:// doi.org/10.1101/2020.12.24.20248802

Frontera, J. A., Sabadia, S., Lalchan, R., Fang, T., Flusty, B., MillarVernetti, P., ... Galetta, S. (2021). A Prospective Study of Neurologic Disorders in Hospitalized Patients With COVID-19 in New York City. Neurology, 96(4):e575-e586. https://doi.org/10.1212/ wnl.0000000000010979

García, L. F. (2020). Immune response, inflammation, and the clinical spectrum of COVID-19. Frontiers in Immunology, 11. https://doi. org/10.3389/fimmu.2020.01441

Garner, P. (2020). For 7 weeks I have been through a roller coaster of ill health, extreme emotions, and utter exhaustion. British Medical Journal Opinion. https://blogs.bmj.com/bmj/2020/05/05/paulgarner-people-who-have-a-more-protracted-illness-need-help-tounderstand-and-cope-with-the-constantly-shifting-bizarre-sympt oms/

Gómez-Mesa, J. E., Galindo-Coral, S., Montes, M. C., \& Muñoz Martin, A. J. (2021). Thrombosis and coagulopathy in COVID-19. Current Problems in Cardiology, 46(3), 100742. https://doi.org/ 10.1016/j.cpcardiol.2020.100742

Graham, E. L., Clark, J. R., Orban, Z. S., Lim, P. H., Szymanski, A. L., Taylor, C., ... Koralnik, I. J. (2021). Persistent neurologic symptoms and cognitive dysfunction in non-hospitalized Covid-19 "long haulers". Annals of Clinical and Translational Neurology, 8(5), 1073-1085. https://doi.org/10.1002/acn3.51350

Hanff, T. C., Harhay, M. O., Brown, T. S., Cohen, J. B., \& Mohareb, A. M. (2020). Is there an association between COVID-19 mortality and the renin-angiotensin system? A call for epidemiologic 
investigations. Clinical Infectious Diseases, 71(15), 870-874. https://doi.org/10.1093/cid/ciaa329

Hoertel, N., Sanchez-Rico, M., Vernet, R., Beeker, N., Jannot, A.S., Neuraz, A., ...Limosin, F. (2021). Association between antidepressant use and reduced risk of intubation or death in hospitalized patients with COVID-19: results from an observational study. Molecular Psychiatry. https://doi.org/10.1038/ s41380-021-01021-4

Hosp, J. A., Dressing, A., Blazhenets, G., Bormann, T., Rau, A., Schwabenland, M., ... Meyer, P. T. (2021). Cognitive impairment and altered cerebral glucose metabolism in the subacute stage of COVID-19. Brain, 144(4), 1263-1276. https://doi.org/10.1093/ brain/awab009

Huang, C., Huang, L., Wang, Y., Li, X., Ren, L., Gu, X., ... Cao, B. (2021a). 6-month consequences of COVID-19 in patients discharged from hospital: a cohort study. Lancet, 397, 220-232. doi: https://doi.org/10.1016/S0140-6736(20)32656-8

Huang, Y., Pinto, M.D., Borelli, J.L. Mehrabadi, M.A., Abrihim, H., Dutt, N., ... Downs, C.A. (2021b). COVID symptoms, symptom clusters, and predictors for becoming a long-hauler: Looking for clarity in the haze of the pandemic. medRxiv 2021.03.03.21252086; doi: https://doi.org/10.1101/2021.03.03. 21252086

Imazio, M., Klingel, K., Kindermann, I., Brucato, A., De Rosa, F. G., Adler, Y., \& De Ferrari, G. M. (2020). COVID-19 pandemic and troponin: indirect myocardial injury, myocardial inflammation or myocarditis? Heart, 106(15), 1127-1131. https://doi.org/10.1136/ heartjnl-2020-317186

Inouye, S. K. (2021). The Importance of Delirium and Delirium Prevention in Older Adults During Lockdowns. JAMA, 325(17), 1779. https://doi.org/10.1001/jama.2021.2211

Komaroff, A. (2020, October 15). The tragedy of long COVID. Harvard Health. https://www.health.harvard.edu/blog/the-tragedy-of-thepost-covid-long-haulers-202010152479

Kotfis, K., Williams Roberson, S., Wilson, J. E., Dabrowski, W., Pun, B. T., \& Ely, E. W. (2020). COVID-19: ICU delirium management during SARS-CoV-2 pandemic. Critical Care, 24(1). https://doi. org/10.1186/s13054-020-02882-x

Kumar, G. K. (2011). Hypoxia. 3. Hypoxia and neurotransmitter synthesis. American Journal of Physiology-Cell Physiology, 300(4). https://doi.org/10.1152/ajpcell.00019.2011

Lambert, N., El-Azab, S. A., Ramrakhiani, N. S., Barisano, A., Yu, L., ... Pinto, M. D. (2021). COVID-19 survivors' reports of the timing, duration, and health impacts of post-acute sequelae of SARS-COV-2 (PASC) infection. 10.1101/2021.03.22.21254026

Lamontagne, S.J., Winters, M.F., Pizzagalli, D.A., \& Olmstead, M.C. (2021). Post-acute sequelae of COVID-19: Evidence of mood \& cognitive impairment. Brain, Behavior, \& Immunity - Health, (17), 100347. https://doi.org/10.1016/j.bbih.2021.100347

Leentjens, J., van Haaps, T. F., Wessels, P. F., Schutgens, R. E., \& Middeldorp, S. (2021). COVID-19-associated coagulopathy and antithrombotic agents-lessons after 1 year. The Lancet Haematology. https://doi.org/10.1016/s2352-3026(21)00105-8.

Lenze, E.J., Mattar, C., Zorumski, C.F., Stevens, A., Schweiger, J., Nicol, G.E., ... Reierson, A.M. (2020). Fluvoxamine vs placebo and clinical deterioration in outpatients with symptomatic COVID-19. JAMA, 324(22), 2292-2300. doi:https://doi.org/10. 1001/jama.2020.22760

Logue, J.K., Franko, N.M., McCulloch, D.J., McDonald, D., Magedson, A., Wolf, C.R., \& Chu, H.Y. (2021). Sequelae in adults at 6 months after COVID-19 infection. JAMA Netw Open, 4(2):e210830. doi:https://doi.org/10.1001/jamanetworkopen. 2021.0830

Lopez, L., Hart, L.H., \& Katz, M.H. (2021). Racial and ethnic health disparities related to COVID-19. JAMA, 325(8), 719-720. doi:https://doi.org/10.1001/jama.2020.26443
Maes, M., Song, C., Lin, A., Bonaccorso, S., Kenis, G., Jongh, R., ... Scharpe, S. (1999). Negative immunoregulatory effects of antidepressants: inhibition of interferon- and stimulation of interleukin-10 secretion. Neuropsychopharmacology, 40(4):370-379. https://doi.org/10.1016/S0893-133X(98)00088-8

Mazza, M.G., Zanardi, R., Palladini, M., Rovere-Querini, P., \& Benedetti, F. (2021). Rapid response to selective serotonin reuptake inhibitors in post-COVID depression. https://doi.org/10.1016/j. euroneuro.2021.09.009

Moghimi, N., Di Napoli, M., Biller, J., Siegler, J.E., Shekhar, R., McCullough, L.D., . . . Divani, A.A. (2021). The neurological manifestations of post-acute sequelae of SARS-CoV-2 infection. Current Neurology and Neuroscience Reports, 21:44. https:// doi.org/10.1007/s11910-021-01130-1

Nasreddine, Z.S., Phillips, N.A., Bédirian, V., Charbonneau, S., Whitehead, V., Collin, I., Cummings, J.L., Chertkow H. (2005). The Montreal Cognitive Assessment, MoCA: a brief screening tool for mild cognitive impairment. Journal of the American Geriatric Society, 53: 695-9. doi:10.1111/j.15325415.2005.53221.x. PMID 15817019

Østergaard, L. (2021). SARS-CoV-2 related microvascular damage and symptoms during and after COVID-19: Consequences of capillary transit-time changes, tissue hypoxia and inflammation. Physiological Reports, 9(3). https://doi.org/10.14814/ phy 2.14726

Patterson, B.K., Francisco, E.B., Yogendra, R., Long, E., Pise, A., Rodrigues, H., ... Mora, J. (2021a). Persistence of SARS CoV-2 S1 protein in CD16+ monocytes in post-acute sequelae of COVID-19 (PASC) up to 15 months post-infection. bioRxiv.06.25.449905; doi: https://doi.org/10.1101/2021.06.25.449905

Patterson, B.K., Guevara-Coto, J., Yogendra, R., Francisco, E.B., Long, E., Pise, A., ... Mora-Rodríguez, R.A. (2021b). Immune-based prediction of COVID-19 severity and chronicity decoded using machine learning. Frontiers in Immunology, 12, 2520. https://doi. org/10.3389/fimmu.2021.700782

Pitharouli, M.C., Hagenaars, S.P., Glanville, K.P., Coleman, J.R.I., Hotopf, M., Lewis, C.M., \& Pariante, C.M. (2021). Elevated $\mathrm{C}$-reactive protein in patients with depression, independent of genetic, health, and psychosocial factors: results from the UK biobank. American Journal of Psychiatry: 178(6):522-529. https://ajp.psychiatryonline.org/doi/10.1176/appi.ajp.2020.20060 947

Puntmann, V. O., Carerj, M. L., Wieters, I., Fahim, M., Arendt, C., Hoffmann, J., ... Nagel, E. (2020). Outcomes of cardiovascular magnetic resonance imaging in patients recently recovered from coronavirus disease 2019 (COVID-19). JAMA Cardiology, 5(11), 1265. https://doi.org/10.1001/jamacardio.2020.3557

Reichenberg, A., Yirmiya, R., Schuld, A., Kruas, T., Haack, M., Morag, A., \& Pollmacher, T. (2001). Cytokine-associated emotional and cognitive disturbances in humans. Archives of General Psychiatry, 58 : 445-452. doi: https://doi.org/10.1001/archpsyc.58.5.445

Rink, C., \& Khanna, S. (2011). MicroRNA in ischemic stroke etiology and pathology. Physiological Genomics, 43(10), 521-528. https:// doi.org/10.1152/physiolgenomics.00158.2010

Rubin, R. (2020). As their numbers grow, COVID-19 "long haulers" stump experts. JAMA, 324(14), 1381. https://doi.org/10.1001/ jama.2020.17709

Schmidt, C. (2021). COVID-19 long haulers. Nature Biotechnology, 39, 908-913. https://www.nature.com/articles/s41587-021-009847.pdf?proof $=\mathrm{t} \% 29$

Tang, Y., Liu, J., Zhang, D., Xu, Z., Ji, J., \& Wen, C. (2020). Cytokine storm in COVID-19: The current evidence and treatment strategies. Frontiers in Immunology, 11. https://doi.org/10.3389/fimmu. 2020.01708

Taquet, M., Geddes, J. R., Husain, M., Luciano, S., \& Harrison, P. J. (2021). 6-month neurological and psychiatric outcomes in 236379 
survivors of COVID-19: a retrospective cohort study using electronic health records. The Lancet Psychiatry, 8(5), 416-427. https://doi.org/10.1016/s2215-0366(21)00084-5

Tay, M. Z., Poh, C. M., Rénia, L., MacAry, P. A., \& Ng, L. F. (2020). The trinity of COVID-19: immunity, inflammation and intervention. Nature Reviews Immunology, 20(6), 363-374. https://doi. org/10.1038/s41577-020-0311-8

Tenforde, M. W., Kim, S. S., Lindsell, C. J., Billig Rose, E., Shapiro, N. I., Files, D. C., ... Wu, M. J. (2020). Symptom duration and risk factors for delayed return to usual health among outpatients with COVID-19 in a multistate health care systems network United States, March-June 2020. MMWR. Morbidity and Mortality Weekly Report, 69(30), 993-998. 10.15585/mmwr.mm6930e1

Toscano, G., Palmerini, F., Ravaglia, S., Ruiz, L., Invernizzi, P., Cuzzoni, M. G., ... Micieli, G. (2020). Guillain-Barré Syndrome associated with SARS-CoV-2. New England Journal of Medicine, 382(26), 2574-2576. https://doi.org/10.1056/nejmc2009191

Varatharaj, A., Thomas, N., Ellul, M. A., Davies, N. W., Pollak, T. A., Tenorio, E. L., ... Plant, G. (2020). Neurological and neuropsychiatric complications of COVID-19 in 153 patients: a UK-wide surveillance study. The Lancet Psychiatry, 7(10), 875-882. https:// doi.org/10.1016/s2215-0366(20)30287-x

Vink, M., \& Vink-Niese, A. (2020). Could Cognitive Behavioural Therapy Be an Effective Treatment for long COVID and post COVID-19 fatigue syndrome? Lessons from the Qure study for Q-Fever fatigue syndrome. Healthcare (Basel, Switzerland), 8(4), 552. https://doi.org/10.3390/healthcare 8040552

World Health Organization (2021a, August 20). WHO coronavirus (COVID-19) dashboard with vaccination data. http://covid19. who.int

World Health Organization (2021b, October 6). A clinical case definition of post-COVI-19 condition by a Delphi consensus. https:// www.who.int/publications/i/item/WHO-2019-nCoV-Post_ COVID-19_condition-Clinical_case_definition-2021.1

Yehia, B.R., Winegar, A., Fogel, R., Fakih, M., Ottenbacher, A., Jesser, C., ... Cacchione, J. (2020). Association of race with mortality among patients hospitalized with coronavirus disease 2019 (COVID-19) at 92 US hospitals. JAMA Netw
Open;3(8):e2018039. doi:https://doi.org/10.1001/jamanetwor kopen.2020.18039

Yuki, K., Fujiogi, M., \& Koutsogiannaki, S. (2020). COVID-19 pathophysiology: A review. Clinical Immunology, 215, 108427. https:// doi.org/10.1016/j.clim.2020.108427

Publisher's note Springer Nature remains neutral with regard to jurisdictional claims in published maps and institutional affiliations.

Rafael A. Rivas-Vazquez, PsyD, is chief neuropsychologist at First Choice Neurology in Miami, Florida. He is adjunct faculty at University of Miami and Nova Southeastern University. He specializes in diagnostic and cognitive assessment of attention-deficit/hyperactivity disorder, sleep disorders, and neurogenerative disorders. More recently, he has been active in assessing and characterizing symptomatic presentation of post-COVID-19 syndrome.

Gustavo Rey, PhD, was head of the Neuropsychology Service at Miami Children's Hospital Brain Institute for 14 years and is now Clinical Associate Professor in the Department of Neurology at the University of Miami Miller School of Medicine. His areas of interest involve the neuro-behavioral correlates of epilepsy, aphasia, traumatic brain injury, and cross-cultural assessment and test development for Hispanic groups in the US.

Alan Quintana, BA, is currently a second-year graduate student in the neuropsychology track at Nova Southeastern University. His clinical and research interests are mild cognitive impairment and Alzheimer's disease.

Dr. Ana A. Rivas-Vazquez has been in clinical practice and has overseen training programs for psychologists for over 40 years. She is currently director of postdoctoral residency at Citrus Health Network and is Chief Wellness Officer at Baptist Hospital of Miami. Throughout the pandemic, she has overseen the physician wellness program at Baptist Hospital, designed to provide support and minimize burn-out for the medical and nursing staff. 